\title{
A SYSTEMATIC REVIEW OF STUDIES ON QUALITY OF LIFE IN CHILDREN WITH CONGENITAL GASTRO-INTESTINAL ANOMALIES
}

\author{
S.V. Glinianaia ${ }^{1}$, N.D. Embleton ${ }^{1,2}$, J. Rankin ${ }^{1}$ \\ ${ }^{1}$ Institute of Health and Society, Newcastle University, ${ }^{2}$ Newcastle Neonatal Service, Royal Victoria \\ Infirmary, Newcastle upon Tyne, UK
}

Background and aims: Few studies have assessed quality of life (QoL) for children born with major congenital anomalies. We aimed to review studies reporting QoL in children born with congenital gastrointestinal anomalies requiring neonatal surgery.

Methods: The inclusion criteria for this review were: studies published in English from 1990 to 2010, using validated instruments for assessment of QoL in children born with congenital diaphragmatic hernia, oesophageal atresia or abdominal wall defects. Literature search, development of the research protocol and data extraction were performed according to standard systematic review methodology.

Results: Of 185 hits, 104 papers were excluded after applying search restrictions and removing duplicates. After scanning 81 abstracts, 32 full-text papers were reviewed, of which 9 were included. All studies measured health-related QoL, none measured subjective wellbeing. Instruments used to assess health-related QoL in children varied considerably. Many studies had major methodological flaws, e.g. from a single institution, retrospective cohorts, low sample size, and/or low follow up rates. There was substantial age variation (children 1-15y). Higher quality papers were from Northern Europe (Netherlands, Norway). Data from the included studies suggest that QoL of children with congenital gastro-intestinal anomalies changes during the life-course and by young adulthood, is comparable with that of the general population.

Conclusion: The reviewed studies consider health status and functioning as a major determinant of QoL. Current concepts of QoL suggest that subjective wellbeing should be an integral part of QoL assessment. Further studies need to include age-adjusted, validated instruments to measure QoL throughout the lifecourse. 